\title{
Schwierige Entwöhnung vom Respirator: Beatmung und weitere Strategien
}

\section{Difficult Weaning}

Autoren

Institute

\section{T. Barchfeld ${ }^{1}$, B. Schönhofer ${ }^{2}$}

Fachkrankenhaus Kloster Grafschaft, Schmallenberg

Abteilung für Pneumologie und internistische Intensivmedizin, Klinikum Region Hannover $\mathrm{GmbH}$, Krankenhaus Oststadt-Heidehaus, Hannover eingereicht 4. 4. 2006

akzeptiert 30.4. 2006

Bibliografie

DOI $10.1055 / \mathrm{s}-2006-932215$

Pneumologie 2006; 60; 616-628

(c) Georg Thieme Verlag KG

Stuttgart · New York

ISSN 0934-8387

Korrespondenzadresse

Prof. Dr. med.

Bernd Schönhofer

Abteilung für Pneumologie und internistische Intensivmedizin

Klinikum Region Hannover

$\mathrm{GmbH}$

Krankenhaus

Oststadt-Heidehaus

30659 Hannover

Bernd.Schoenhofer@t-online.de

Serienherausgeber

D. Köhler, Schmallenberg

B. Schönhofer, Hannover

\section{Zusammenfassung}

Das ventilatorische Versagen infolge Überlastung und/oder reduzierter Kapazität der Atempumpe ist die häufigste Ursache für die erfolglose Entwöhnung vom Respirator (Weaning) und damit für die Notwendigkeit der invasiven Langzeitbeatmung (LZB). Dabei steht die chronisch obstruktive Lungenerkrankung (COPD) im Vordergrund. Der wichtigste klinische Parameter für die erschöpfte Atemmuskulatur ist die schnelle flache Atmung („rapid shallow breathing“). Weitere Faktoren, die im Weaning-Prozess den Erfolg negativ beeinflussen können, sind neuromuskuläre Erkrankungen, Herzinsuffizienz, die inadäquate tiefe und lange Sedierung, Mikro- bzw. Makroaspiration, Mangelernährung, Anämie und Adipositas per magna. Protokollbasierte Entwöhnungsstrategien und die Verwendung von so genannten „Weaning-Prädiktoren“ sind hilfreich. Dennoch ist der erfahrene Intensivmediziner im Weaning nicht zu ersetzen. Das Ziel im Weaning nach LZB heißt „Rekonditionierung der Atemmuskulatur". Alle therapeutischen Maßnahmen sollten darauf abzielen, durch entlastende Strategien eine Erholung der überlasteten Atemmuskulatur zu erreichen. Assistierte Beatmungsformen sind im Weaning weit verbreitet. Eine ausreichende Entlastung der erschöpften Atemmuskulatur lässt sich damit sehr häufig nicht erreichen, da die inspiratorische Atemarbeit signifikant erhöht bleibt. Demgegenüber führt eine individuell adaptierte, kontrollierte Beatmung (mit Druckoder Volumenvorgabe), die auch bei klarem Bewusstsein des Patienten möglich ist, zur maximalen Entlastung der Atemmuskulatur und deren Erholung. Weitere entlastende Maßnahmen sind die Korrektur einer Anämie, die pharmakologische Reduktion des Atemantriebes (z.B. durch Morphinpräparate), die Sauerstoffgabe während der Spontanatmung, das Aufrichten des Oberkörpers vor allem bei Adipositas und

\section{Abstract}

Respiratory failure as a result of overload and/or reduced capacity of the respiratory muscles is the most common cause of unsuccessful weaning and the need for long term mechanical ventilation. Chronic obstructive pulmonary disease (COPD) is the most common underlying cause leading into long term mechanical ventilation. The most important clinical parameter for fatigue of the respiratory muscles is the rapid shallow breathing index. Other essential factors which impact weaning failure, are the underlying diseases (e.g. neuromuscular disease or heart failure), micro- and macro aspiration, malnutrition, anemia and obesity. A protocol based strategy to discontinue mechanical ventilation and the use of weaning predictors are helpful. Nonetheless the experienced physician is irreplacable in the weaning process. Reconditioning of the respiratory muscles is the main focus during weaning after long term mechanical ventilation and all therapeutic measures should be targeted to unload the fatiguing respiratory muscles. With the widely used assisted ventilation modes, the inspiratory work of breathing is still significantly increased. Only controlled mechanical ventilation (pressure- or volume controlled), which may also be applied to unsedated patients when individually adapted, offers the best possible relief and recovery of the respiratory muscles. Additional strategies, such as the balancing of anemia, reduction of the respiratory drive with i.e. morphine derivates, oxygen therapy during spontaneous-breathing trials and supine position for patients with obesity contribute to the recovery. Particularly patients with chronic lung diseases with hypercapnia benefit from the use of non invasive ventilation (NIV) after extubation to prevent postextubation failure and even after tracheostomy. However, NIV should only be applied under close monitoring and in cooperative pa- 
die Überführung des katabolen in den anabolen Ernährungszustand. Insbesondere bei Patienten mit broncho-pulmonalen Vorerkrankungen (z. B. COPD) hat die nichtinvasive Beatmung (NIV) während der Entwöhnung von der invasiven Beatmung, aber auch in der Postextubationsphase einen hohen Stellenwert. NIV sollte im Weaning-Prozess unter engmaschigem Monitoring der Vitalfunktionen, bei vorhandener Kooperation des Patienten und mit Wissen um die Grenzen der Methode durchgeführt werden. Sterben am Respirator in der Endphase eines chronischen Krankheitsverlaufes bleibt eine große Herausforderung für alle Beteiligten. Gelingt ein erfolgreiches „Weaning in der Terminalphase“, dann ist es manchem Patienten doch noch möglich, den letzten Lebensabschnitt außerhalb einer Intensivstation zu verbringen.

\section{Hintergrund}

Bei der Mehrzahl der beatmungspflichtigen Patienten kann die maschinelle Unterstützung bei ausreichender Spontanatmung oft kurzfristig beendet werden. Dies gilt für Patienten nach einem operativen Eingriff wie für Patienten in der nicht-chirurgischen Intensivmedizin, die infolge einer akuten Erkrankung kurzfristig beatmet werden müssen. Demgegenüber gestaltet sich die Beatmung bei chronisch kranken, häufig multimorbiden Patienten mit fortgeschrittenen Lungen- oder Herzerkrankungen in der operativen wie nichtoperativen Intensivmedizin als äußerst schwierig und es kommt nicht selten zur Langzeitbeatmung (LZB) [1]. Obwohl eine allgemein akzeptierte Definition fehlt, ist es sinnvoll, nach 14-21 Tagen kontinuierlicher Beatmung von „Langzeitbeatmung“ zu sprechen [2,3]. Zur LZB kommt es in etwa $10 \%$ der Beatmungsfälle [2], wobei mindestens $50 \%$ der Ressourcen einer Intensivstation dadurch verbraucht werden [4]. Ebenso kommt es zur LZB bei Patienten mit schweren Akuterkrankungen und langen, komplikationsreichen intensivmedizinischen Aufenthalten, in deren Folge andere Begleiterkrankungen oder die intensivmedizinische Behandlung selbst Ursache einer Langzeitbeatmung werden können (z.B. tients, always considering the limits of the method. Dying under mechanical ventilation in the end stage illness is still a challenge for all involved persons. In the end stage of their disease for some patients it is possible to discontinue mechanical ventilation so they can spend the last period of their lives on a normal ward or even at home.

Tab. 1 Charakteristika und Weaning - Outcome im Vergleich unterschiedlicher Zentren modifiziert nach [7], weiterführende Literatur [103, 104, 105, 106]; ALI: Acute lung injury

\begin{tabular}{|c|c|c|c|c|c|}
\hline $\begin{array}{l}\text { Erstautor und Lite- } \\
\text { raturstelle }\end{array}$ & Nava & Gracey & Scheinhorn & Bagley & Schönhofer \\
\hline Patientenzahl & 42 & 132 & 421 & 278 & 232 \\
\hline $\begin{array}{l}\text { durchschn. Alter } \\
\text { (Jahre) }\end{array}$ & 67 & 67 & 70 & 67 & 65 \\
\hline \multicolumn{6}{|l|}{ Diagnosen } \\
\hline COPD (\%) & 100 & $13 \%$ & $24 \%$ & $30 \%$ & $54 \%$ \\
\hline Chirurgie (\%) & - & $63 \%$ & $24 \%$ & $11 \%$ & $7 \%$ \\
\hline $\operatorname{ALI}(\%)$ & - & $1 \%$ & $32 \%$ & $28 \%$ & $5 \%$ \\
\hline neuromuskulär (\%) & - & - & $8 \%$ & $19 \%$ & $16 \%$ \\
\hline \multicolumn{6}{|l|}{ Weaning } \\
\hline $\begin{array}{l}\text { Beatmung auswärts } \\
\text { (Tage) }\end{array}$ & 13 & 14 & 49 & - & 44 \\
\hline $\begin{array}{l}\text { Weaningdauer im } \\
\text { Zentrum (Tage) }\end{array}$ & 30 & 16 & 39 & - & 7,5 \\
\hline Weaningerfolg (\%) & $54 \%$ & $70 \%$ & $53 \%$ & $38 \%$ & 65 \\
\hline \multicolumn{6}{|l|}{ Überleben } \\
\hline bei Entlassung (\%) & $71 \%$ & $90 \%$ & $71 \%$ & $53 \%$ & $72 \%$ \\
\hline Langzeit (\%) & $\begin{array}{c}55 \% \\
(1 \mathrm{Jahr})\end{array}$ & - & $\begin{array}{l}28 \% \\
(1 \mathrm{Jahr})\end{array}$ & - & $\begin{array}{l}63,7 \% \\
\text { (3 Monate) }\end{array}$ \\
\hline
\end{tabular}




\section{Pathophysiologie des schwierigen Weaning}

Das Ungleichgewicht zwischen Last und Kapazität der Atemmuskulatur ist die häufigste Ursache für ein schwieriges Weaning (siehe Artikel Pathophysiologische Grundlagen der mechanischen Beatmung in dieser Serie [10]). Am Beispiel der COPD lässt sich veranschaulichen, dass im Mittelpunkt der Pathogenese einer schwierigen Entwöhnung die erschöpfte Atempumpe mit einem Ungleichgewicht zwischen der muskulären Überlastung (d.h. erhöhter Atemarbeit) und der reduzierten muskulären Kapazität (d.h. verminderter Kraft und Ausdauer der Atemmuskulatur) steht [11]. Die vermehrte Atemarbeit (work of breathing: WOB) wird vorwiegend inspiratorisch geleistet und besteht im Wesentlichen aus einer resistiven Komponente (dominierend bei COPD) und einer elastischen Komponente (dominierend Adipositas per magna, ARDS, Lungenödem, Lungenfibrose, Torsionsskoliose oder Z.n. Lungenparenchym-resezierenden OPs). In selteneren Fällen können auch Störungen des zentralen Atemantriebs (z.B. Hirnstamminfarkte) Gründe für eine fehlende Spontanatmung sein.

Überschreitet die Belastung der Atemmuskulatur etwa 40\% der möglichen Kraft (so genannte Erschöpfungsschwelle), kommt es zur muskulären Erschöpfung [11,12], sichtbar an der Hyperkapnie. In diesem Zusammenhang ist festzuhalten, dass vor allem das ventilatorische Versagen mit dem Leitwert Hyperkapnie und konsekutiver respiratorischer Azidose, jedoch deutlich seltener das hypoxämische Lungenversagen die führende Ursache für die erfolglose Entwöhnung vom Respirator und LZB ist $[11,13]$. Die nahezu völlige Erschöpfung der Atemmuskulatur, gemessen z.B. anhand eines erhöhten „Tension-Time-Index“, erwies sich als entscheidende Ursache des erfolglosen Weanings $[13,14]$. Neben der vermehrten Last kann die reduzierte Kapazität der Atemmuskulatur Ursache der fehlenden Entwöhnbarkeit vom Respirator sein. Klassisches Beispiel für Erkrankungen, die mit einer reduzierten Kapazität der Atemmuskulatur einhergehen, ist die Muskelschwäche infolge der neuromuskulären Erkrankungen. Diese können in angeborene (Spinale Muskelatrophien, Muskeldystrophien) und erworbene (Amyotrophe Lateralsklerose, Post-Poliomyelitissyndrom, Frühsommer-Meningoenzephalitis etc.) sowie in progrediente und reversible Erkrankungen unterschieden werden. Die Lungenüberblähung bei schwergradiger COPD führt ebenfalls zur Reduktion der Muskelkapazität.

Das klinische Korrelat der drohenden Erschöpfung der Atemmuskulatur ist u.a. die schnelle flache Atmung (rapid shallow breathing) [15] mit der Folge der Erhöhung der Totraumventilation. Es resultiert eine Hypoventilation, die an der $\mathrm{CO}_{2}$-Retention zu erkennen ist [12]. Neben der erschöpften Atemmuskulatur führen Critical Illness Polyneuropathie bzw. Myopathie, hochdosierte Steroidtherapie über längere Zeit, Sepsis und bestimmte Beatmungsformen (d.h. Ventilator-induziert) zur morphologisch nachweisbaren Schädigung des Zwerchfells und damit zur Langzeitbeatmung.

\section{Weitere Ursachen für schwieriges Weaning}

\section{Aspiration}

Patienten mit Tracheotomie werden häufig sowohl während der Spontanatmungsphasen als auch während der Beatmung oral ernährt. Hierbei besteht die Gefahr der Makroaspiration, aber auch einer Mikroaspiration, die häufig weder von den betreuen-

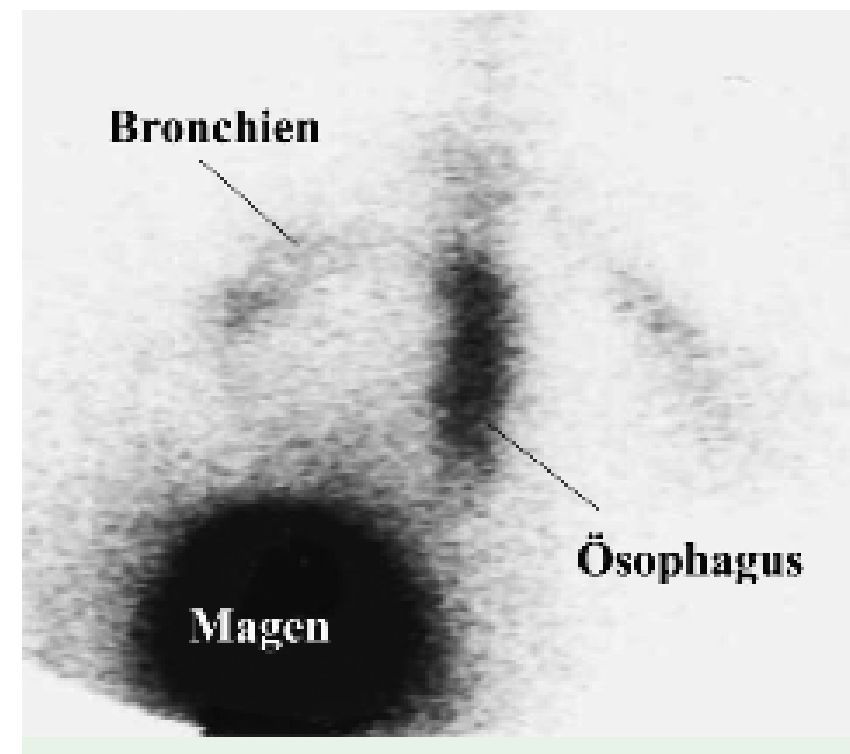

Abb. 1 Nuklearmedizinischer Nachweis einer Aspiration mit Technetium in beide Hauptbronchien.

den Pflegekräften noch vom Patienten selber bemerkt wird. In der Abb. 1 ist eine typische, vom Patienten unbemerkte Aspiration von nuklearmedizinisch markierten Nahrungsmitteln dargestellt. Aspiration ist oft auch durch eine geblockte Trachealkanüle nicht zu verhindern. Im Gegenteil, die geblockte Kanüle kann den physiologischen Schluckakt behindern und die Aspiration so begünstigen. Auch bei Ernährung über eine nasogastrale Sonde ist die Aspiration von Nahrungsmitteln infolge gastroösophagealem Reflux nicht ausgeschlossen [16]. Eine PEG senkt hingegen die Aspirationsgefahr deutlich [17]. Die kontinuierliche subglottische Sekretabsaugung (Continuous aspiration of subglottic secretions; CASS) führte zwar in einer In-vivo-Studie am Tiermodell zur Abnahme der bakteriellen Kolonisation, gleichzeitig kam es aber zu einer deutlichen Schädigung der Trachealschleimhaut [18]. Ohne weiteres lassen sich diese Ergebnisse nicht auf den klinischen Alltag übertragen.

\section{Malnutrition}

Mangelernährung und reduzierte Muskelmasse („fat free mass“ und geringer body mass index (BMI) $<20 \mathrm{~kg} / \mathrm{m}^{2}$ ) sind sensitive und relevante Ernährungsparameter, die häufig bei beatmeten COPD- und Emphysem-Patienten vom Typ „pink puffer“ angetroffen werden [19]. An Laborparametern wird häufig bei diesen Patienten ein vermindertes Serumalbumin als auch Phosphat-, Kalzium- und Magnesiummangel beobachtet. Diese Mangelzustände können die Entwöhnung von der Beatmung erschweren.

\section{Anämie}

Anämie unterschiedlicher Genese ist ein häufiges Problem beim langzeitbeatmeten Patienten [20]. Die zugrunde liegenden Ursachen hierfür sind vielgestaltig (häufige Blutabnahmen, latente gastrointestinale Blutung und chronische Inflammation mit Suppression der Blutbildung). Neben der CRIT-study [21] haben zwei weitere Arbeiten [22,23] gezeigt, dass eine liberalere Transfusionspraktik mit einer höheren Mortalität verknüpft sein kann. Diese Arbeiten halten eine Erythrozytensubstitution erst ab einem Hämoglobinwert von $7 \mathrm{~g} / \mathrm{dl}$ bei Akut-Erkrankten für notwendig. Der Zusammenhang zur erhöhten Mortalität bei Erythrozytensubstitution bestand nach diesen Studien in dem 
erhöhten Nachweis von Bakteriämien und erhöhtem Risiko für eine Ventilator-assoziierte Pneumonie. Als Limitation dieser Arbeiten muss angeführt werden, dass nur Patienten in der „frühen" Phase der Beatmung (Tag 1-5) beobachtet wurden. Langzeitbeatmete Patienten waren nicht Gegenstand dieser Studien. Die CRIT-study war lediglich eine Beobachtungsstudie, so dass nur eine Assoziation und kein kausaler Zusammenhang beschrieben wird. Kürzlich ergab die Auswertung einer Datenbank mit 2524 COPD-Patienten, dass Anämie zur Verschlechterung der Prognose in diesem Patientenkollektiv führt [24]. Vor allem bei Patienten mit chronischen Lungenerkrankungen, wie z.B. schwergradiger COPD, führt die mit der Anämie einhergehende Reduktion des Sauerstoff-Gehaltes bzw. der Sauerstoff-Transportkapazität sekundär zum erhöhten Ventilationsbedarf und damit zu einer gesteigerten Atemarbeit. Letzteres führt zur weiteren Überlastung der Atemmuskulatur und kann ein entscheidender Grund für die fehlende Entwöhnbarkeit vom Respirator sein $[25,26]$. Zum pathophysiologischen Hintergrund des Sauerstoffgehaltes siehe auch „Pathophysiologische Grundlagen der mechanischen Beatmung“ [10].

\section{Linksherzinsuffizienz}

Der Zusammenhang von Hämodynamik und Ventilation ist komplex, aber entscheidend, um die Pathophysiologie des langzeitbeatmeten Patienten mit Linksherzinsuffizienz oder besser Globalherzinsuffizienz zu verstehen. Nicht selten ist die Linksherzinsuffizienz, z.B. infolge einer koronaren Herzerkrankung, wesentliche Ursache für eine erfolglose Entwöhnung vom Respirator [27].

Die Spontanatmung des Herzinsuffizienten geht atemabhängig mit hohen intrathorakalen Druckschwankungen und gesteigerte WOB mit erhöhtem $\mathrm{O}_{2}$-Bedarf der Atemmuskulatur (so genannte „Oxygen cost of breathing“) einher. Hierbei kann es im Sinne eines „Steal-Effektes“ zur kritischen Myokardischämie kommen. Umgekehrt nimmt der $\mathrm{O}_{2}$-Verbrauch der Atemmuskulatur mit Beginn der maschinellen Beatmung wieder ab und gleichzeitig nehmen der $\mathrm{O}_{2}$-Transport zum Myokard und die LV-Funktion zu. Es ließ sich mittels Einschwemmkatheter zeigen, dass es als Ausdruck der progredienten LV-Insuffizienz während der Spontanatmung im Vergleich zur Beatmung zum Anstieg des pulmonal-kapillären Verschlussdruckes kam [28].

Bei chronisch Herzkranken, die nicht vom Respirator entwöhnbar waren, war ein unzureichender Anstieg des Herzzeitvolumens während der Spontanatmungsphase Ausdruck einer reduzierten kardialen Leistungsreserve [29].

\section{Sedierung und neuropsychiatrische Aspekte}

Delirante Zustände, Entzugssymptomatik und komplexe Angststörungen sind häufig bei langzeitbeatmeten Patienten zu beobachten [30]. Sie sind oft die Folge von längerer Behandlung mit Sedativa. Eine unnötig lange oder tiefe Sedierung kann die Beatmungszeit und den Krankenhausaufenthalt deutlich verlängern [31]. Häufig angeführte Gründe für die Sedierung sind inakzeptable Agitation oder „Fighting“ des Patienten am Beatmungsgerät. Nicht selten sind fehlende Entwöhnungskonzepte, aber auch der pflegerische Personalmangel Ursachen für die prolongierte Sedierung $[32,33]$. Bedingt durch exogene Faktoren wie Licht und Lärm ist der Schlaf von Intensivpatienten häufig fragmentiert, der Tag-Nachtrhythmus gestört; der Tiefschlaf ist vermindert oder fehlt ganz. Die Folgen von Übersedierung und Schlafentzug sind häufig anhaltende delirante Zustände, die nur schwer zu handhaben sind und sich negativ verstärken können
[34]. In einer strukturierten Erhebung der Lebensqualität nach schwierigem Weaning und intensivmedizinischer Behandlung berichteten $67 \%$ der Patienten von weiter bestehenden Schlafstörungen [35]. Eine aktuelle Befragung ehemaliger Intensivpatienten ergab bei $30 \%$ der Befragten persistierende Alpträume, $14 \%$ fühlten sich davon psychisch beeinträchtigt [36].

\section{Adipositas}

Proportional zur wachsenden Prävalenz in unserer Bevölkerung sind Patienten mit Adipositas per magna zunehmend intensivbzw. beatmungspflichtig und schwer vom Respirator entwöhnbar. Adipositas beeinträchtigt die atemmechanischen Eigenschaften der Lunge, des Zwerchfells und des knöchernen Thorax vor allem in Rückenlage, was die WOB unmittelbar erhöht [37].

- Abb. 2 zeigt die Unterschiede der Atemarbeit bei Adipositas zwischen liegender und halbsitzender Position bei Spontanatmung.

\section{Entwöhnungsstrategie bei der schwierigen Entwöhnung}

Beatmungszentren mit dem Schwerpunkt „Respiratorentwöhnung“ haben im Laufe der vergangenen Jahre Entwöhnungsstrategie mit dem Ziel konzipiert, langzeitbeatmete Patienten erfolgreich vom Respirator zu entwöhnen. Auch wenn evidenzbasierte Untersuchungen hierzu bisher weitgehend fehlen, spricht die relativ hohe Erfolgsrate der Zentren für diese Rationale ( Tab. 1). Im Folgenden werden einzelne Aspekte unserer Strategie erläutert.

\section{Bedeutung der Prädiktoren}

Bei einer unkomplizierten Beatmung z.B. nach einer Operation ist die klinische Erfahrung des Behandlungsteams nach wie vor der entscheidende Faktor, insbesondere beim Wiedereinsetzen der Spontanatmung des Patienten und der Entscheidung über die Extubation. Insbesondere bei Patienten mit chronischen Krankheitsbildern bzw. akuten Erkrankungen bei bestehenden schweren Lungen- oder Herzerkrankungen (Multimorbidität)

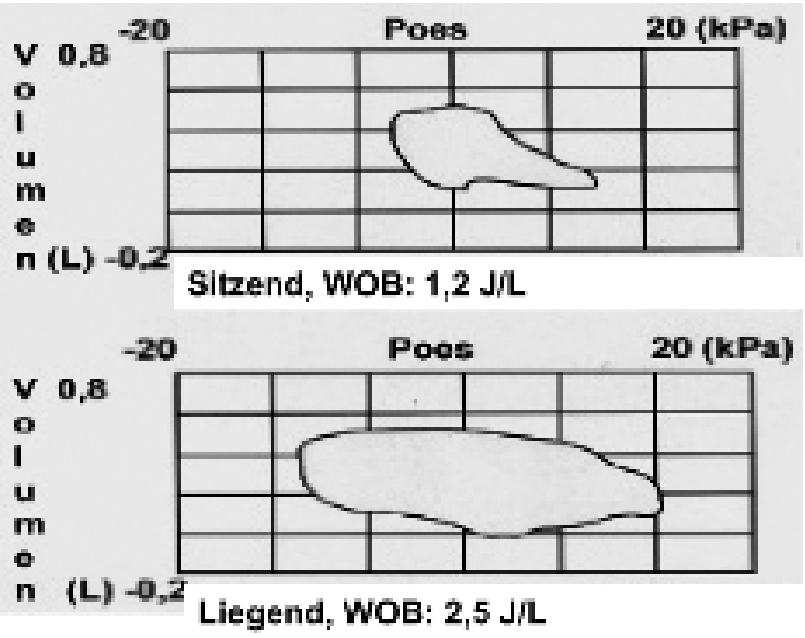

Abb. 2 Atemarbeit (WOB) bei Patienten mit Adipositas permagna, Messung der WOB im Sitzen versus Liegen, Poes: Ösophagealer Druck. 
kann es jedoch aufgrund subjektiver Fehleinschätzung der Gesamtsituation durch das Behandlungsteam zur unnötigen Verzögerung der Entwöhnung vom Respirator kommen. Andererseits geht die Reintubation (im Sinne des Extubationsfehlers) mit Zunahme von Morbidität und Mortalität einher [38]. Aufgrund dieser Unsicherheit wird seit längerem nach unterschiedlichen Parametern zur Vorhersage von Erfolg und Misserfolg des Weanings (so genannten „Prädiktoren“) gesucht, um im Beatmungs- bzw. Entwöhnungsmanagement mehr Sicherheit zu haben [39]. Hierbei stehen univariate Parameter (z.B. Atemfrequenz, Atemzugvolumen, Atemminutenvolumen, Vitalkapazität, Mundverschlussdruck „P0,1“ oder inspiratorische Muskelkraft „Pi $\mathrm{i}_{\max }$ “) und multivariate Indices (z.B. Ratio aus Atemfrequenz und Tidalvolumen: „Rapid shallow breathing Index“) zur Verfügung. Insbesondere den univariaten Parametern kann im schwierigen Entwöhnungsprozess als Entscheidungshilfe zur Diskonnektion eines Patienten vom Respirator eine klinische Relevanz zukommen. Vor allem in spezialisierten Abteilungen sind einige Parameter inzwischen fester Bestandteil von WeaningStrategien, Protokollen und Computer-gestützten Konzepten. Häufig können Prädiktoren die komplexe Situation eines individuellen Beatmungspatienten nicht adäquat erfassen, so dass der erfahrene Kliniker die wichtige Entscheidung bzgl. WeaningKonzept und Extubationszeitpunkt treffen muss. Im klinischen Alltag der schwierigen Entwöhnung vom Respirator befinden wir uns oft im Spannungsfeld zwischen Prädikt-basierter und erfahrungsgeprägter Strategie, entsprechend der Formulierung von Milic Emili „Weaning - Kunst oder Wissenschaft“ [40]. Entwöhnungsstrategien, die den Verlauf einfach zu messender Parameter (z. B. Atemfrequenz und Atemzugvolumen) berücksichtigen, verkürzen die Beatmungszeit und verbessern den Entwöhnungserfolg und andere Outcome-Parameter [15, 39,41,42]. Wir empfehlen daher, einfach zu messende Prädiktoren zu erheben und diese in die Entwöhnungskonzepte der Intensivstation zu implementieren.

\section{T-piece trial}

In der Beurteilung der Entwöhnbarkeit vom Respirator kommt dem so genannten „T-Stück-Versuch“ (T-piece-trial) in der klinischen Praxis als auch in wissenschaftlichen Untersuchungen eine besondere Bedeutung zu. Während des T-piece-trial atmet der Patient spontan $\left(\mathrm{O}_{2}\right.$-Insufflation und gleichzeitige Befeuchtung der Atemluft ohne maschinelle Atemunterstützung). Dabei werden die oben genannten Parameter bzw. Indices qualitativ oder quantitativ ermittelt. Die Fähigkeit zur Spontanatmung sowie der Verlauf der erhobenen Messparameter führen zu mehr Sicherheit bei der Frage der Extubation. Da nachgewiesen wurde, dass sich Spontanatmungsphasen von 30 Minuten und 120 Minuten Dauer bzgl. Beurteilung der Entwöhnbarkeit nicht unterscheiden, ist aus Gründen der Praktikabilität die kürzere Beobachtungsdauer zu empfehlen [43].

\section{Spontanatemphasen: T-Stück versus assistierte \\ Spontanatmung}

Im Falle einer Tracheotomie spielt die Gestaltung der Spontanatemphasen die entscheidende Rolle im weiteren erfolgreichen Fortschreiten des Weaning-Prozesses. Bei langzeitbeatmeten Patienten kommen überwiegend assistierende Beatmungsverfahren wie z.B. pressure support ventilation (PSV) zur Anwendung $[3,41]$. Zweifelsohne wird die inspiratorische WOB durch assistierte Beatmung deutlich reduziert [44]. Es ließ sich jedoch z.B. anhand von EMG-Registrierungen des Zwerchfells bei assis- tierenden Beatmungsverfahren zeigen, dass relevante WOB nicht nur zum Zeitpunkt des eigentlichen Triggervorgangs, sondern auch während der sich anschließenden maschinell unterstützten Inspiration geleistet wird [45-47]. Diese bei der assistierten Beatmung vom Patienten aufzubringende WOB wird häufig unterschätzt, da sie exakt nur mit größerem technischen Aufwand invasiv (z.B. mit transdiaphragmaler Druckmessung) zu erfassen ist. Weitverbreitete Beatmungsformen in der Entwöhnung vom Respirator sind Pressure Support Ventilation (PSV) und synchronisierte intermittierende mandatorische Spontanatmung (SIMV) [48]. Bei der SIMV wird die Atemfrequenz schrittweise reduziert. Mehrere Arbeiten haben belegen können, dass die Atemarbeit nicht nur bei den spontanen Atemzügen, sondern auch bei getriggerten Atemzügen zunimmt, da das Atemzentrum sich nicht so schnell an die intermittierende Druckunterstützung adaptieren kann $[46,49,50]$.

PSV unterstützt die Spontanatmung des Patienten mit einem frei wählbaren positiven Druck [51]. Jeder Atemzug wird vom Patienten getriggert und im Idealfall dauert die Druckunterstützung in der Inspiration an, bis der Flow des Patienten abfällt und in die Exspiration umschaltet. Jubran und Mitarbeiter konnten zeigen, dass Patienten mit COPD unter PSV bereits in der späten Inspirationsphase exspiratorische Muskelaktivität aufwiesen [52]. Dies kann zur Asynchronität von Patient und Beatmungsgerät führen, was im Weaning-Prozess kontraproduktiv ist. Dagegen besteht bei langzeitbeatmeten Patienten mit erschöpfter Atemmuskulatur das Ziel der Beatmung darin, eine maximale Reduktion der WOB zu erreichen. Bei der kontrollierten Beatmung ist die Reduktion des $\mathrm{O}_{2}$-Verbrauchs der Atemmuskulatur und damit die Erholung der erschöpften Muskulatur am höchsten [53]. Es konnte gezeigt werden, dass die kontrollierte Beatmung (mit Druck- oder Volumenvorgabe, zunächst kontinuierlich zur Rekonditionierung, im weiteren Verlauf mit Ausdehnung der Spontanatmung über T-Stück) in mehr als 50\% der Fälle bei Patienten mit LZB binnen weniger Tage zur erfolgreichen Entwöhnung führt [54]. Eigene Untersuchungen zur Effektivität der kontrollierten Beatmung bei chronisch ventilatorischer Insuffizienz ergaben, dass unterschiedliche Beatmungsmodi, z.B. Volumenvorgabe und Druckvorgabe, zur Besserung der Spontanatmung führen [55].

Die Anwendung von kontrollierter Beatmung über längere Zeit führt im Tierversuch zur Atrophie der Atemmuskulatur [56,57]. Inzwischen liegen auch Erkenntnisse zu molekularbiologischen Mechanismen vor, die dem Ventilator-induzierten diaphragmalen Schaden zugrunde liegen [58]. Diese Ergebnisse beziehen sich allerdings auf initial gesunde Muskulatur und sind daher nicht ohne weiteres auf das genannte Patientenkollektiv mit erschöpfter Atemmuskulatur übertragbar. Für unser Konzept der kontrollierten Beatmung zur maximalen Entlastung der erschöpften Atmungsmuskulatur ist entscheidend, dass der Patient intermittierend spontan atmet (d.h. Entlastung versus Belastung). Der Erfolg dieser Methode ist natürlich auch von der vorliegenden Grunderkrankung abhängig.

\section{Invasiver Beatmungszugang: Beatmungstubus - Tracheotomie}

Dem Tubus als Beatmungszugang kommt bei der schwierigen Entwöhnung eine wichtige Bedeutung zu. Der Trachealtubus führt proportional zur Länge zu erhöhter resistiver WOB [59]. (Hagen-Poiseuille-Gesetz: Strömungswiderstand umgekehrt proportional zur 4. Potenz des Tubusdurchmessers). Durch Sekretablagerung kommt es zu einer weiteren Verengung des Strö- 
mungsquerschnitts und damit Steigerung des Widerstands [60]. Um zusätzliche tubusbedingte resistive WOB $\mathrm{zu}$ vermeiden, kann zur Kompensation des Widerstands je nach Innendurchmesser eine Druckunterstützung von 5-8 mbar eingestellt werden. Bei Patienten mit hohem Atemantrieb und hohen inspiratorischen Gasflüssen ist diese Druckunterstützung häufig unzureichend. Auch wenn der tubusbedingte Widerstand durch die in manchen Beatmungsgeräten angebotene flussgesteuerte automatische Tubuskompensation (ATC) theoretisch ausgeglichen werden kann, konnte bisher der praktische Nutzen dieser Option nicht überzeugend gezeigt werden [61]. Daher gilt die Empfehlung, bei Problemen im Weaning kaliberstarke Tuben, z.B. mit Innendurchmesser von $9 \mathrm{~mm}$, zu verwenden.

Einige Argumente sprechen dafür, dass die Tracheotomie bei länger dauernder Beatmung der Beatmungszugang der Wahl ist. Es besteht eine große Varianz von möglichen Vorgehensweisen bzgl. Indikation, Zeitpunkt und Technik der Tracheotomie. Wesentliche Vorteile der Tracheotomie im Vergleich zum oral oder nasal applizierten Tubus bestehen, neben der möglichen Einsparung von oder sogar Beendigung der Sedierung, in der Reduktion der resistiven WOB [62]. Im Wesentlichen stehen zwei Tracheotomietechniken zur Verfügung: die chirurgisch angelegte und die Punktionstracheotomie. Aufgrund der einfachen technischen Handhabung und der geringeren Komplikationsrate hat sich die Punktionstracheotomie gegenüber dem chirurgisch angelegten Tracheostoma zunehmend durchgesetzt [63,64,65]. Es war lange klinische Praxis, den Patienten nach einer Beatmungsdauer von etwa 14 Tagen zu tracheotomieren [66]. Mit der Etablierung der Punktionstracheotomie und dem ökonomischen Anreiz durch das DRG(Diagnose related groups-)Abrechnungssystem verkürzt sich der Zeitraum zwischen Intubation und Tracheotomie [67]. Auch wenn vergleichende Untersuchungen noch fehlen, werden mit der Frühtracheotomie mögliche Vorteile der nicht-invasiven Beatmung (NIV, siehe nächsten Abschnitt) als Entwöhnungsmaßnahme unmittelbar nach früher Extubation nicht ausreichend genutzt.

Beim erfolgreich entwöhnten tracheotomierten Patienten, bei dem die pulmonale Situation kritisch bleibt und/oder infolge weiter bestehender ventilatorischer Insuffizienz die Indikation zur NIV über Maske besteht, kann die Übergangszeit mittels „Platzhalter“ (Abb. 3) (international „Button“ genannt) überbrückt werden [68]. Dieses Hilfsmittel reicht nur bis zur ventralen Trachealwand, verschließt den Tracheotomiekanal, kann als Zugang zum Absaugen und Bronchoskopie genutzt werden und ermöglicht das Sprechen, einen effektiven Hustenstoß und die NIV. Der Vorteil des Platzhalters besteht darin, dass der Tracheostomakanal zwar verschlossen ist, aber nicht schrumpft und als Beatmungszugang im Falle einer erneuten Indikation zur invasiven Beatmung weiterhin zur Verfügung steht. Es sei hier kurz angemerkt, dass der Platzhalter entweder mangels Erfahrung des Behandlungsteams oder infolge ungünstiger Anatomie nicht immer regelrecht positioniert werden kann. In der Regel muss die „Trachealplatte“ individuell zugeschnitten werden.

Bereits während der Planung eines Tracheostomas ist die Option des späteren Verschlusses zu bedenken. Die immer noch häufigen so genannten „definitiven“ chirurgisch angelegten Tracheostomata erweisen sich im späteren Verschlussmanagement oft als problematisch, da sie im Gegensatz zur Punktionstracheotomie kaum Schrumpfungstendenz aufweisen. Bei tracheotomierten Patienten mit Indikation zur häuslichen Beatmung nach erfolgreichem Weaning, bei denen es nicht zum spontanen Verschluss des Tracheostomas kommt, sondern eine plastisch-chi- rurgische Deckung notwendig ist, muss geprüft werden, ob sie postoperativ eine mehrtägige NIV-Pause ohne das Auftreten einer bedrohlichen ventilatorischen Insuffizienz tolerieren. Das Ziel dieser Therapiepause ist, potenzielle NIV-bedingte Komplikationen wie z.B. Nahtdehiszenz, Mediastinal- oder Hautemphysem zu verhindern.

\section{Protokollbasierte Strategien}

Prinzipiell können protokollbasierte Strategien zu einer Verbesserung des Entwöhnungserfolges führen. In diesem Zusammenhang wurde kürzlich überzeugend gezeigt, dass ca. 30\% der zuvor in nicht spezialisierten Intensivstationen langzeitbeatmeten Patienten kurz nach Übernahme ins Weaning-Zentrum definitiv vom Respirator entwöhnt waren, nachdem sie dort im Rahmen des protokollbasierten T-piece-trials längere Spontanatmungsphasen tolerierten [69].

In einer Untersuchung von Ely u. Mitarb. prüfte ein Physiotherapeut jeweils am Morgen anhand von fünf Parametern die Fähigkeit der Patienten, über einen längeren Zeitraum spontan zu atmen [42]. Der behandelnde Arzt wurde über das Testergebnis informiert und legte anschließend die weitere therapeutische Vorgehensweise fest. Im Vergleich zur Kontrollgruppe ohne definiertes Konzept war die Beatmungszeit in der Interventionsgruppe signifikant kürzer (4,5 Tage versus 6 Tage, $p=0,003)$. Außerdem führte dieses Protokoll zur Reduktion von Komplikationen, Reintubationsrate, Hospitalisationstagen und Behandlungskosten.

In einer anderen Studie führte die morgendliche Unterbrechung der Sedierung (im Vgl. zu kontinuierlicher Sedierung) zur Verkürzung der Beatmungszeit und der Aufenthaltsdauer auf der Intensivstation [70].

Protokollbasierte Entwöhnungsstrategien können unter bestimmten Umständen erfolgreich eingesetzt werden. Es bleibt kritisch anzumerken, dass sie den erfahrenen Intensivmediziner als zentrale Größe im komplizierten Entwöhnungsprozess nicht ersetzen können. In einer Untersuchung der Johns Hopkins University (Baltimore) ergab der Vergleich zwischen einem Behandlungsteam, das sich durch ein hohes Qualitätsniveau und gute personelle Ausstattung auszeichnete, aber keine Weaning-Protokolle einsetzte, und einer protokollbasierten Entwöhnungsstrategie keinen Unterschied bzgl. der Rate der erfolgreich entwöhnten Patienten [71].

\section{Weitere Maßnahmen zur Entlastung und Rekondi- tionierung der erschöpften Atemmuskulatur}

Neben den bisher beschriebenen Beatmungsprinzipien sind weitere nicht-maschinelle Therapieoptionen feste Bestandteile des Weaning-Konzeptes bei LZB (siehe Tab. 2).

\section{Reduktion der Atemarbeit (WOB)}

Es wurde bereits weiter oben auf den Zusammenhang zwischen Anämie und Atemarbeit, insbesondere bei langzeitbeatmeten Patienten mit schwergradiger COPD, hingewiesen. Durch Transfusion von Erythrozyten-Konzentraten wird der $\mathrm{O}_{2}$-Gehalt bzw. -Transport verbessert. Wir konnten für das COPD-Kollektiv im Vergleich zu lungengesunden Patienten mit Anämie zeigen, dass Atemminutenvolumen und Atemarbeit durch Anheben des HB-Wertes von 9,5 g\% auf etwa $12 \mathrm{~g} \%$ um ca. $25 \%$ reduziert wurde und damit im Einzelfall die erfolgreiche Respiratorentwöhnung mithilfe der Transfusion erreicht werden konnte [26]. Trotz 

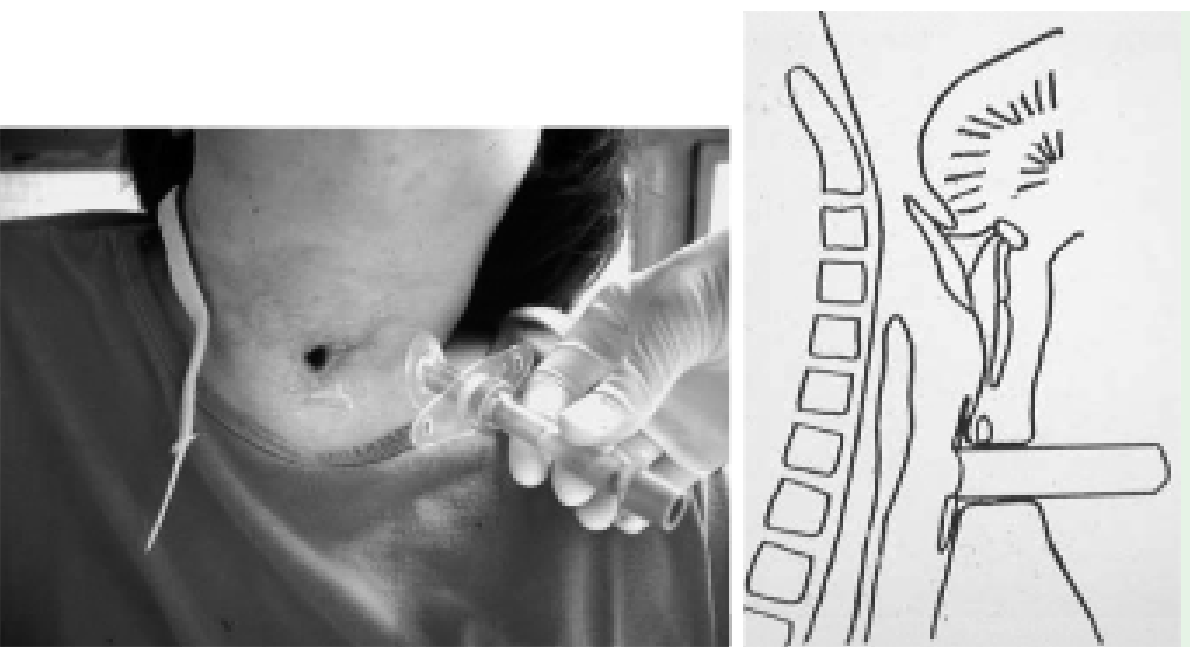

Abb. 3 a Punktionstracheotomie mit Platzhalter, b Schematische Darstellung der Funktionsweise.

der kontroversen Diskussion zum Stellenwert der Bluttransfusion in der Intensivmedizin [72] gehört die Korrektur der Anämie in der besagten Patientengruppe inzwischen zum festen Bestandteil des Entwöhnungskonzeptes. Für Patienten mit LZB infolge COPD empfehlen wir einen Hämoglobinwert von mindestens $12 \mathrm{~g} / \mathrm{dl}$ anzustreben (siehe unten). Neben dem Ausgleich der Anämie kann eine Entlastung der Atemmuskulatur, d.h. Reduktion der WOB, auch in bestimmten Fällen durch pharmakologische Reduktion des Atemantriebes z.B. durch Morphinpräparate, Sauerstoffgabe während der Spontanatmung und aufrechte Körperposition vor allem bei adipösen Patienten erreicht werden $[73,74]$.

\section{Verbesserung des Ernährungszustandes und Metabolismus}

Unterschiedliche Faktoren können bei langzeitbeatmeten Patienten zur Mangelernährung und damit zur Kachexie und muskulären Schwäche beitragen. Häufig besteht z. B. bei schwergradiger COPD Mangelernährung [75]. Darüber hinaus können chronische Inflammation, Hormondefizite und Immobilität den Ernährungszustand und den Stoffwechsel negativ beeinflussen. Zum Thema „Ernährungstherapie bei LZB“ liegen nur wenige klinisch relevante Daten vor. Eine ausreichende Gabe von Kalorien (d.h. $2400-3000 \mathrm{kcal} / \mathrm{d}$ ) sowie ausgeglichene Bilanz der Elektrolyte und Mineralien sind jedoch wesentliche Voraussetzungen für eine erfolgreiche Entwöhnung [76]. Die Ernährung und damit die Verteilung der Kalorienträger bei Patienten mit respiratorischer Insuffizienz sollte bei ca. 35-40\% Kohlenhydraten $(\mathrm{KH}), 10-15 \%$ Eiweiß und 50-55\% Fett liegen. Eine übermäßige Zufuhr von KH zur Deckung des Kalorienbedarfs sollte vermieden werden, da hierbei eine Zunahme der $\mathrm{CO}_{2}$-Produktion zu einer vermehrten Ventilation führt, welche bei langzeitbeatmeten Patienten mit überlasteter Atemmuskulatur kontraproduktiv ist (Belastung der schon überlasteten Atempumpe). Auch wenn die Genese der „Critical-Illness-Polyneuropathie“ multifaktoriell ist, wird das Überangebot an $\mathrm{KH}$ als ein wichtiger pathogenetischer Faktor diskutiert [77]. Möglicherweise haben die bisher zur Therapie der pulmonalen Kachexie untersuchten pharmakologischen Therapieansätze, wie z. B. Wachstumshormone, Antioxidanzien, antiinflammatorische Pharmaka und Anabolika in Kombination mit supplementärer Ernährung [78-81] ebenfalls Einfluss auf den Entwöhnungserfolg. Deren klinische Relevanz muss in wissenschaftlichen Studien noch weiter untersucht werden.
Tab. 2 Nicht-maschinelle Behandlungsoptionen im Weaningkonzept

\begin{tabular}{l} 
Ziel: Atemarbeit. Reduktion der bronchialen Obstruktion \\
Muskulatur \\
Beta-2 Inhalation \\
\hline Schleimhaut \\
Adrenalin \\
Steroide \\
Antibiotika Inhalation + Instillation \\
Sekret-Clearance \\
physikalische Therapie \\
Husten ermöglichen (Platzhalter, NIV) \\
Sedation vermeiden \\
\hline Ziel: Atemarbeit. Reduktion der Ventilation/Last \\
\hline O-Gehalt erhöhen \\
Blutgabe \\
(Sauerstoff) \\
\hline kontrollierte Beatmung \\
(am wachen Patienten) \\
großer oraler Tubus \\
\hline Lagerung \\
Hochlagerung, besonders bei OHS
\end{tabular}

Der Stellenwert des frühzeitigen Wechsels von parenteraler Ernährung auf die gastrointestinale Nahrungszufuhr bei Patienten mit LZB scheint vorteilhaft, insbesondere zur Prävention der Critical-Illness-Polyneuropathie. Beim frühzeitigen Wechsel können Katheter-assoziierte Infektionen eher verhindert werden. Nasogastrale Sonden können aber ein unabhängiger Risikofaktor für die Entwicklung einer Ventilator-assoziierten Pneumonie sein [16]. Hierbei könnte die frühzeitige Anlage einer perkutanen Gastrostomie eine Alternative sein. Eine erste kontrollierte Studie [17] mit einer kleinen Fallzahl von 20 Patienten mit neurologischen Defiziten und Beatmungspflichtigkeit konnte einen signifikanten Vorteil der PEG-Sonde gegenüber der nasogastralen Sonde bezüglich der Anzahl von Ventilator-assoziierten Pneumonien nachweisen. Um den Stellenwert der PEG-Sonde und dem Zeitpunkt der Anlage zu evaluieren, sind aber größere kontrollierte Studien bei Patienten mit LZB erforderlich.

\section{Physiotherapie}

Auch wenn die evidenzbasierte Datenlage dürftig ist, hat die Physiotherapie inzwischen im Entwöhnungskonzept der Patienten mit LZB einen hohen Stellenwert.

Die Standardverfahren der Physiotherapie wie passives Durchbewegen und Sekretmobilisation mit herkömmlichen Verfahren 
wie Perkussion, Vibrax etc. sind für die Patienten mit Langzeitbeatmung ungenügend. Die Physiotherapie muss möglichst frühzeitig in den Prozess der Entwöhnung integriert werden. Der Beginn von spezialisierter Physiotherapie bei Erreichen von 12 Stunden Spontanatmung ist eindeutig zu spät. Neben der Auswahl des Kanülenaufsatzes zur Spontanatmung (feuchte Nase, Sprechventil, Dauer-PEEP) spielt die differenzierte Therapie zur Sekretmobilisation eine entscheidende Rolle bei effizienter Physiotherapie. Prinzipiell muss unter Berücksichtigung der Grunderkrankung (obstruktiv: i.d.R. instabile Atemwege bei vorhandener Exspirationskraft/neuromuskulär: i.d.R. stabile Atemwege bei fehlender Exspirationskraft) eine Unterstützung des ineffizienten Hustenstoßes zur effektiven Sekretmobilisation erfolgen ( Abb.4). Ist der Patient noch intubiert, so ist der Hustenstoß infolge fehlenden Glottisschlusses selbst bei ausreichender Kraft der Muskulatur ineffektiv. Hinzu kommt, dass die meisten Weaning-Patienten infolge der Grunderkrankung (z.B. COPD) keine oder nur eine sehr unzureichende mukoziliäre Clearance haben.

Bei instabilen Atemwegen kommen je nach Exspirationskraft unterschiedliche Hilfsmittel (z.B. positive expiratory pressure: PEP; Cornet, Flutter, etc.) zur Anwendung ( Abb. 5). Bei neuromuskulär erkrankten Patienten lässt sich die fehlende Exspirationskraft durch andere Hilfsmittel (Cough-Assist, Air Stacking Manöver oder manuelle Unterstützung) erreichen, um Sekret zu mobilisieren. Trotz dieses differenzierten Vorgehens, das auf pathophysiologischen Erkenntnissen beruht, fehlen hierzu weitgehend kontrollierte Studien. Der besondere Stellenwert der Physiotherapie im Entwöhnungsprozess kommt auch dadurch zum Ausdruck, dass die European Respiratory Society (ERS) eine „task force“ zum Thema „Physiotherapy for adult patients with critical illness" und die Deutsche Gesellschaft für Pneumologie (DGP) einen Ausbildungsgang zum Atmungstherapeuten mit einem Schwerpunkt „Weaning“ eingerichtet haben [82].

\section{Antipsychotische und anxiolytische Therapiekonzepte} Die Behandlung von deliranten Zuständen und Agitationszuständen bei Patienten mit Langzeitbeatmung erfordert viel Geduld, pflegerische und ärztliche Erfahrung und Umgebungsbedingungen der Intensivstation, die einer psychischen Stabilisierung förderlich sind. Das so genannte „Intensivstation-Delirium“ oder auch „Psychodrama“ ist ein eigenständiger Risikofaktor für einen längeren Krankenhausaufenthalt, eine höhere Mortalität und höhere Behandlungskosten [83]. Es ist sehr schwierig, delirante, agitierte und angstgezeichnete Patienten, die beatmet sind, psychisch zu stabilisieren [84]. Nach LZB und länger dauernder Sedierung erleben viele Patienten eine regelrechte Entzugssymptomatik. Effektive Betreuung dieser Patienten erfordert eine ausreichende personelle Besetzung vor allem im Bereich der Pflegekräfte, um eine undifferenzierte und nicht indizierte Gabe von Psychopharmaka zu vermeiden. Nicht selten ist zusätzlich eine Kombination aus initial hochdosierter antipsychotischer und anxiolytischer Pharmakotherapie indiziert. Scores zur Erfassung der Agitation und Steuerung der Sedierung sind hilfreich $[34,85]$. Auf die Einhaltung des Tag-Nacht-Rhythmus muss geachtet werden. Darüber hinaus können Agitationszustände auch durch die fehlende Kommunikationsfähigkeit verursacht sein, so dass alle Optionen zur Verbesserung der
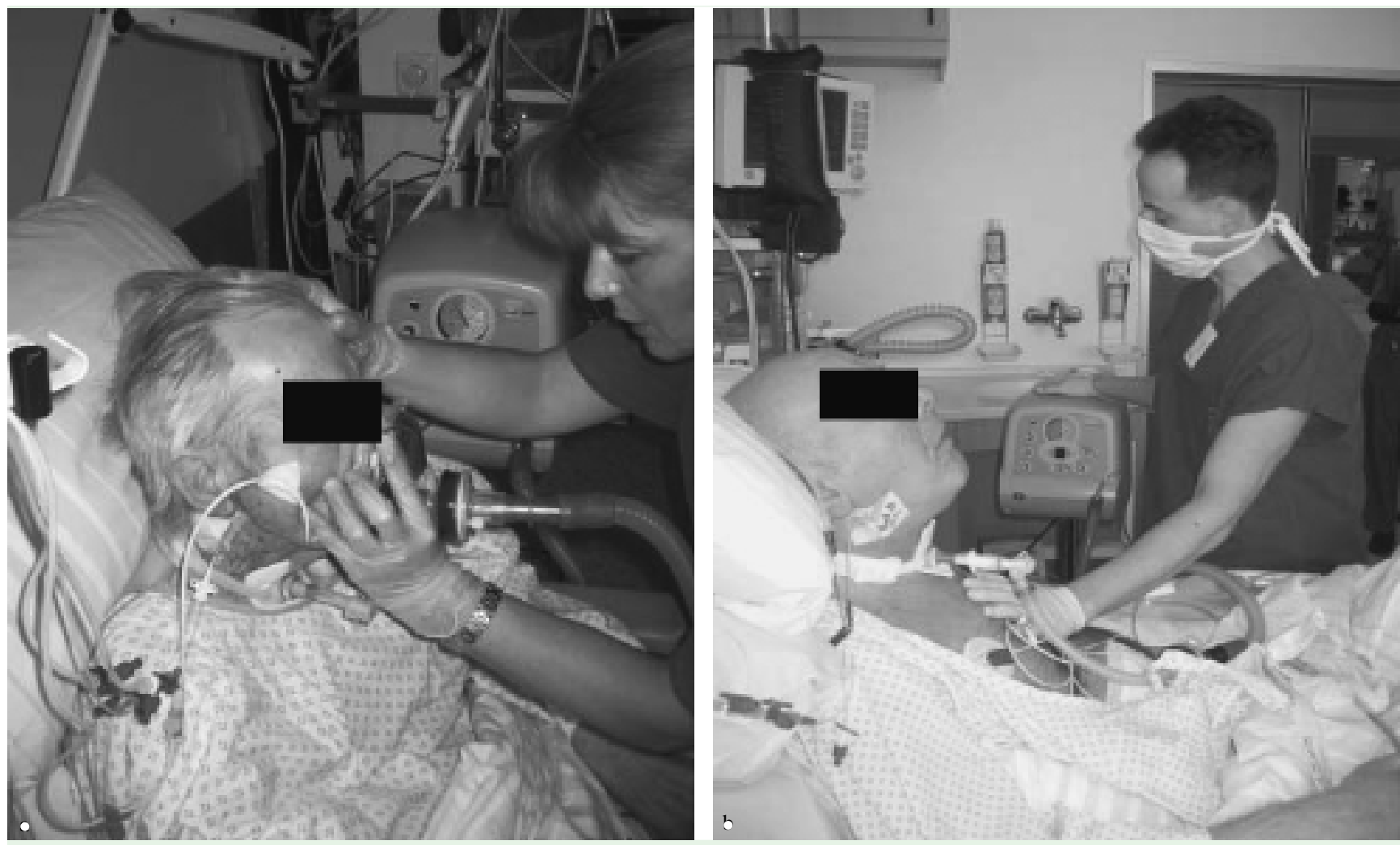

Abb. 4 Cough Assist (maschinelle Hustenunterstützung) zur Sekretmobilisation bei neuromuskulären Patienten; a via Maske und b via invasiven Beatmungszugang. 


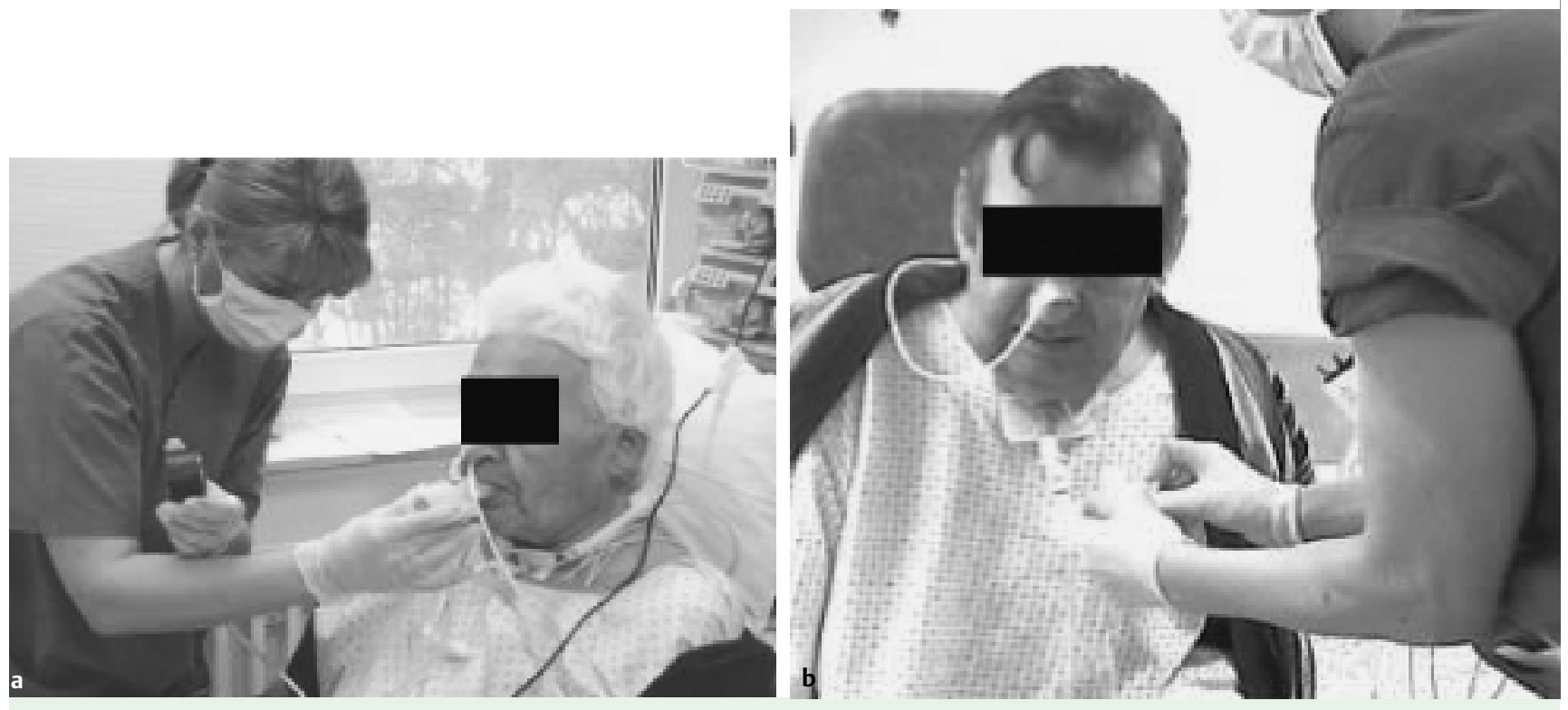

Abb. 5 Sekretmobilisation bei obstruktiven Patienten mit Hilfe von PEP; a via Mundstück und b via Trachealkanüle.

Kommunikation genutzt werden müssen. So lassen sich auch andere Ursachen der Agitation (wie z.B. Schmerzen) eruieren und beseitigen. Nach Möglichkeit sollten die Angehörigen in das Behandlungskonzept in Form von individuell adaptierten Besuchszeiten eingebunden werden. Im Einzelfall ist die konsiliarische Mitbetreuung durch einen in der Beatmung erfahrenen Psychiater sinnvoll.

\section{NIV in der Entwöhnung vom Respirator und Post-Extubationsphase}

Bei dieser Thematik ist die Unterscheidung zwischen hyperkapnischem und hypoxämischem Atmungsversagen bezüglich des Stellenwertes der NIV zu beachten.

Bei invasiv beatmeten Patienten mit schwergradiger COPD lässt sich die Erfolgsrate der Respiratorentwöhnung durch frühzeitige Extubation und unmittelbar anschließende NIV, verglichen mit einer invasiv beatmeten Kontrollgruppe, signifikant verbessern. Zusätzlich kommt es hierdurch zur Reduktion der Letalitäts- sowie Reintubations- und Tracheotomierate [86,87].

Demgegenüber kann der frühzeitige Einsatz von NIV im Weaning von Patienten mit hypoxämischem Atmungsversagen bei zurzeit unzureichender Studienlage nicht generell empfohlen werden, auch wenn die Ergebnisse einer unkontrollierten Studie mit kleiner Fallzahl zur Frühextubation und anschließender NIV bei Nicht-COPD-Patienten günstige Effekte auf physiologische Parameter wie Blutgase, Atemmuster, Atemarbeit und Shuntfraktion zeigte [88].

In der Post-Extubationsphase hat die NIV in der Prävention, aber auch Therapie einer erneuten akut respiratorischen Insuffizienz einen hohen Stellenwert. Bei der Anwendung der NIV in der Postextubationsphase müssen folgende Prinzipien gelten: nicht auf das Postextubationsversagen warten, mit adäquaten Beatmungsdrücken (in Bezug auf die Grunderkrankung) beatmen und die Grenzen der Methode kennen. Das Dilemma der Reintubation infolge erneuter ventilatorischer Insuffizienz besteht darin, dass sie mit einer hohen Komplikations- und Letalitätsrate verbunden ist [38]. Vor allem bei Risikopatienten mit COPD und
Hypersekretion, die nach Extubation eine hyperkapnische Atmungsinsuffizienz entwickeln, führt der frühzeitige Einsatz von NIV zur Reduktion der Reintubations- und Letalitätsrate [89-92].

Beim LZB-tracheotomierten Patienten kann der Übergang von der invasiven zur nicht-invasiven Beatmung meist mit dem Erreichen von 8-10 h Spontanatmung erreicht werden. (Stellenwert des Platzhalters s.o.) Trotz allem ist es nicht möglich, alle Patienten zu diesem Zeitpunkt nicht-invasiv zu beatmen. Anhaltende Durchgangssyndrome, fortgeschrittenes Alter und erheblich eingeschränkte Kooperation machen die Durchführung einer effektiven NIV mitunter unmöglich. Bei diesen Patienten ist ein erheblich längeres Zeitfenster zur Entwöhnung mit anschließender Frührehabilitation notwendig, um schließlich doch noch eine erfolgreiche Entwöhnung zu erreichen. Trotz aller Maßnahmen bleiben je nach Patientenkollektiv und Weaning-Erfahrung $30-40 \%$ der Patienten dauerhaft beatmet bzw. versterben zeitnah infolge der Schwere der Grunderkrankung (siehe unten).

Besteht bei Patienten nach erfolgreichem Weaning vom Respirator weiterhin eine chronisch ventilatorische (d.h. hyperkapnische) Insuffizienz, dann profitieren viele von NIV in Form der intermittierenden Selbstbeatmung (sog. „häusliche Beatmung“) [54]. Gegen einen unselektiven Einsatz der NIV bei akuter respiratorischer Insuffizienz (ARI) in der Postextubationsphase sprechen aktuelle Ergebnisse randomisierter und kontrollierter Studien, in denen sich entweder kein Unterschied zwischen der NIV und der Standardtherapie zeigte [93] oder das NIV-Kollektiv sogar eine signifikant erhöhte Reintubations- und Letalitätsrate aufwies [94]. In beiden Studien wurden vorwiegend Patienten mit hypoxämischer ARI und deutlich weniger Patienten mit COPD eingeschlossen. Trotz der methodischen Mängel der Studien (wie z.B. verzögerter Beginn der NIV, unzureichender Beatmungseffekt, geringe Beatmungsdrücke, unzureichende Erfahrung des Teams und hohe Reintubationsrate) sind diese Ergebnisse ernst zu nehmen, weil sie in gewisser Weise den klinischen Alltag in der Breite der Patientenversorgung widerspiegeln. Ferrer und Mitarbeiter zeigten kürzlich auch für ein spezialisiertes Team, dass NIV bei Patienten mit hypoxämischer Atmungsinsuffizienz nach Extubation zu keiner Verbesserung der Outcomepa- 
rameter führt [92]. Es lässt sich also schlussfolgern, dass langjährige Erfahrung des Teams, frühzeitiger Beginn mit NIV, engmaschiges Monitoring der Vitalfunktionen und Blutgasanalyse und die unverzügliche Reintubation bei Hinweisen auf Atmungsinsuffizienz unverzichtbare Voraussetzungen für den Einsatz von NIV in der Postextubationsphase sind.

\section{Das Beatmungszentrum}

An dieser Stelle sei auf den Artikel in dieser Serie „Ort der maschinellen Beatmung im Beatmungszentrum - Intensivstation, Intermediate care oder spezialisierte Normalstation“ [95] verwiesen. Erwähnt werden soll an dieser Stelle jedoch, dass der respiratorischen Intermediärstation (respiratory intermediate care unit) im Weaningzentrum die Schlüsselrolle zukommt [9]. Bei adäquatem Personalschlüssel, hoher Qualifikation des Teams und guter technischer Ausrüstung kann die respiratorische Intermediärstation einen wichtigen Anteil der bisherigen Aufgaben der Intensivstation effektiv und kostengünstig übernehmen. Nicht zu unterschätzen ist schließlich die Bedeutung der assoziierten Normalstation, da sich hier der Zustand der vom Respirator entwöhnten Patienten konsolidieren muss und ggf. die Heimbeatmung begonnen wird.

\section{Lebensqualität nach Entwöhnung vom Respirator}

In jüngerer Vergangenheit sind mehrere Studien zur Lebensqualität nach Beatmung in unterschiedlichen Patientenkollektiven publiziert worden $[96,97]$. In unserer eigenen Untersuchung zur Lebensqualität nach erfolgreichem Weaning erwies sich die zur ventilatorischen Insuffizienz führende Diagnose als entscheidende Determinante der Lebensqualität [98]. Bei Patienten mit Thorakorestriktion war die Lebensqualität besser als bei Patienten mit neuromuskulären Erkrankungen und COPD. Trotz der signifikanten somatischen Beeinträchtigung der Patienten war deren mentale Verfassung nur geringgradig beeinträchtigt.

\section{Definitiv nicht entwöhnbare Patienten im Finalstadium}

Ein gewisser Anteil langzeitbeatmeter Patienten ist auch im spezialisierten Beatmungszentrum trotz konsequenter Anwendung der genannten Strategien und Konzepte nicht entwöhnbar oder die ventilatorische Situation ist als so instabil einzuschätzen, dass binnen Tagen bis Wochen eine erneute Beatmungspflichtigkeit droht [99]. In einer Untersuchung unseres Patientenkollektives über einen Zeitraum von 10 Jahren misslang bei $32 \%$ der Patienten die Entwöhnung definitiv [54]. Die Ergebnisse eines britischen Weaning-Zentrums ergaben, dass 35\% der Patienten nicht zu entwöhnen waren und $27 \%$ der Patienten verstarben [6]. Bei bleibender Respiratorabhängigkeit in dieser Lebensphase werden die meisten Patienten abhängig von den örtlichen Gegebenheiten in heimatnahen Krankenhäusern oder sonstigen, meistens nicht spezialisierten Einrichtungen (wie z. B. Pflegeheime) untergebracht. Zunehmend etablieren sich ambulante Pflegedienste, die eine Betreuung der Patienten in ihrer häuslichen Umgebung anbieten. Unter sozialen Aspekten ist dies wünschenswert. Aber nicht jeder langzeitbeatmete Patient lässt sich außerhalb einer Intensivstation betreuen. Darüber hinaus ist die
Betreuung eines beatmeten Patienten in seinem häuslichen Umgebung ohne relevanten Beitrag des sozialen Umfeldes sehr kostenintensiv (Dreischichtbetrieb mit Kosten von ca. 10000 bis 15000 Euro pro Monat). Im Sinne der Verteilungsgerechtigkeit stellt sich hier die Frage; wer kann wie, wo und in welchem Umfang betreut werden? Berücksichtigt man die demographische Entwicklung unseres Landes wird dieser Themenkomplex in $\mathrm{Zu}$ kunft noch schwieriger werden.

Infolge der Schwere der Erkrankung ist es in jedem Falle notwendig, den Patientenwillen zu berücksichtigen. Häufig wird hier klar der Wunsch geäußert, die Therapiemaßnahmen zu begrenzen. In diesem Kontext ist die intensive Kommunikation mit den Angehörigen ein wesentlicher Beitrag, um die komplexe Gesamtsituation des Patienten (medizinisch, psychosozial, ethisch und juristisch) aufzuarbeiten. Dies gilt insbesonders in Fällen, in denen nach einer langen intensivmedizinischen Therapie der Patientenwille nicht $\mathrm{zu}$ eruieren ist (z.B. durch ein anhaltendes Durchgangssyndrom). Hierbei muss zwischen Therapiebegrenzung (im Englischen „Withholding“) und Therapieabbruch (im Englischen „Withdrawing“) unterschieden werden. Therapiebegrenzung bedeutet, dass prinzipiell mögliche ärztliche Maßnahmen bei klinischer Verschlechterung aufgrund einer infausten Prognose des Patienten (z.B. Dialyse bei Nierenversagen) nicht mehr erfolgen. Therapieabbruch bedeutet im Zusammenhang mit diesem Artikel, dass die Beatmung in der Intensität reduziert und schließlich beendet wird. Diese Problematik wird ausführlich in dem Artikel zur Ethik in der Beatmungsmedizin in dieser Serie diskutiert [100]. Mit der Diskonnektion vom Beatmungsgerät ist jedoch nicht zwangsläufig das unmittelbar eintretende Sterben des Patienten verbunden. Bei einer Minderheit dieser Patienten kommt es nach Therapieabbruch wider Erwarten sogar zur längerfristigen klinischen Stabilisierung [101].

Manchmal gelingt es, einem im Finalstadium befindlichen Langzeitbeatmeten das spontane Atmen und Sprechen ohne starke Dyspnoeempfindung zu ermöglichen (d.h. „Weaning in der Terminalphase“). Patienten sind so häufiger in der Lage, den letzten Lebensabschnitt auf Normalstation, in pflegerischen Einrichtungen oder im Idealfall in häuslicher Umgebung zu verbringen [54]. Da die Mehrheit der Patienten jedoch im unmittelbaren zeitlichen Zusammenhang mit dem Therapieabbruch auf der Intensivstation oder assoziierten Stationen stirbt, sollte hier im Idealfall ein gesonderter Bereich vorhanden sein, wo dem Patienten im Beisein seiner Angehörigen ein würdiges Sterben ermöglicht wird. Es existieren abhängig vom kulturellen Umfeld in der Literatur unterschiedliche Empfehlungen zum pharmakologischen Management des „terminalen Weaning“ [102]. In den USA werden teilweise konkrete Anweisungen zum Umgang mit Opiaten, Sedativa und Barbituraten umgesetzt. Auch wenn vergleichbare Protokolle im Europäischen Raum nicht weit verbreitet sind, gilt auch hier, dass Dyspnoe, Agitation und Schmerzen durch Gabe von potenten Analgosedativa konsequent zu therapieren sind. Es ist zu empfehlen, dass die Beatmung nicht abrupt „abgeschaltet“, sondern der Grad der maschinellen Unterstützung im Sinne der Deeskalation allmählich reduziert wird. In Kombination mit der genannten Medikation kommt es dann präfinal häufig zur Hyperkapnie und Azidose und einer Art „intrinsischen Sedierung“. 


\section{Literatur}

1 Gillespie DJ, Marsh HM, Divertie MB et al. Clinical outcome of respiratory failure in patients requiring prolonged (greater than 24 hours) mechanical ventilation. Chest 1986; 90: 364-369

2 Epstein SK, Vuong V. Lack of influence of gender on outcomes of mechanically ventilated medical ICU patients. Chest 1999; 116: $732-$ 739

3 Esteban A, Frutos F, Tobin MJ et al. A comparison of four methods of weaning patients from mechanical ventilation. Spanish Lung Failure Collaborative Group. N Engl J Med 1995; 332: 345 - 350

4 Cohen IL, Booth FV. Cost containment and mechanical ventilation in the United States. New Horiz 1994; 2: 283 - 290

5 Indihar FJ, Forsberg DP. Experience with a prolonged respiratory care unit. Chest 1982; 81: 189-192

6 Pilcher DV, Bailey MJ, Treacher DF et al. Outcomes, cost and long term survival of patients referred to a regional weaning centre. Thorax 2005; 60: 187-192

7 Schönhofer B, Haidl P, Kemper P et al. Entwöhnung vom Respirator („Weaning“) bei Langzeitbeatmung. Dtsch Med Wochenschr 1999; 124: $1022-1028$

8 Corrado A, Roussos C, Ambrosino $N$ et al. Respiratory intermediate care units: a European survey. Eur Respir J 2002; 20: 1343 - 1350

9 Schönhofer B. Respiratory high-dependency units in Germany. Monaldi Arch Chest Dis 1999; 54: 448-451

10 Köhler D, Pfeifer M, Criée C. Pathophysiologische Grundlagen der mechanischen Beatmung. Pneumologie 2006; 60: 100 - 110

11 Vassilakopoulos T, Zakynthinos S, Roussos C. Respiratory muscles and weaning failure. Eur Respir J 1996; 9: 2383-2400

12 Schönhofer B, Köhler D. Ventilatorische Insuffizienz und hyperkapnische Kompensation infolge chronisch belasteter „Atempumpe“. Dtsch Med Wochenschr 1994; 119: 1209-1214

13 Jubran A, Tobin MJ. Passive mechanics of lung and chest wall in patients who failed or succeeded in trials of weaning. Am J Respir Crit Care Med 1997; 155: 916 - 921

14 Vassilakopoulos T, Zakynthinos S, Roussos C. The tension-time index and the frequency/tidal volume ratio are the major pathophysiologic determinants of weaning failure and success. Am J Respir Crit Care Med 1998; 158: $378-385$

15 Yang KL, Tobin MJ. A prospective study of indexes predicting the outcome of trials of weaning from mechanical ventilation. $\mathrm{N}$ Engl J Med 1991; 324: 1445 - 1450

16 Leal-Noval SR, Marquez-Vacaro JA, Garcia-Curiel A et al. Nosocomial pneumonia in patients undergoing heart surgery. Crit Care Med 2000; 28: 935 - 940

17 Kostadima E, Kaditis AG, Alexopoulos EI et al. Early gastrostomy reduces the rate of ventilator-associated pneumonia in stroke or head injury patients. Eur Respir J 2005; 26: 106-111

18 Berra L, Marchi L De, Panigada $M$ et al. Evaluation of continuous aspiration of subglottic secretion in an in vivo study. Crit Care Med 2004; 32: $2071-2078$

19 Cano N, Roth H, Court-Ortune I et al. Nutritional depletion in patients on long-term oxygen therapy and/or home mechanical ventilation. Eur Respir J 2002; 20: 30 - 37

20 Corwin HL, Parsonnet KC, Gettinger A. RBC transfusion in the ICU. Is there a reason? Chest 1995; 108: $767-771$

21 Corwin HL, Gettinger A, Pearl RG et al. The CRIT Study: Anemia and blood transfusion in the critically ill-current clinical practice in the United States. Crit Care Med 2004; 32: 39- 52

22 Shorr AF, Jackson WL, Kelly KM et al. Transfusion practice and blood stream infections in critically ill patients. Chest 2005; 127: 17221728

23 Gong MN, Thompson BT, Williams $P$ et al. Clinical predictors of and mortality in acute respiratory distress syndrome: potential role of red cell transfusion. Crit Care Med 2005; 33: 1191 - 1198

24 Similowski T, Agusti A, MacNee W et al. The potential impact of anaemia of chronic disease in COPD. Eur Respir J 2006; 27: 390 - 396

25 Schönhofer B, Wenzel M, Geibel $M$ et al. Blood transfusion and lung function in chronically anemic patients with severe chronic obstructive pulmonary disease. Crit Care Med 1998; 26: 1824-1828

26 Schönhofer B, Bohrer H, Kohler D. Blood transfusion facilitating difficult weaning from the ventilator. Anaesthesia 1998; 53: 181-184

27 Srivastava S, Chatila W, Amoateng-Adjepong Yet al. Myocardial ischemia and weaning failure in patients with coronary artery disease: an update. Crit Care Med 1999; 27: 2109-2112
28 Lemaire F, Teboul JL, Cinotti L et al. Acute left ventricular dysfunction during unsuccessful weaning from mechanical ventilation. Anesthesiology 1988; 69: 171 - 179

29 Jubran A, Mathru M, Dries D et al. Continuous recordings of mixed venous oxygen saturation during weaning from mechanical ventilation and the ramifications thereof. Am J Respir Crit Care Med 1998; 158: $1763-1769$

30 Ely EW, Margolin R, Francis J et al. Evaluation of delirium in critically ill patients: Validation of the Confusion Assessment Method for the Intensive Care Unit (CAM-ICU). Crit Care Med 2001; 29: 1370-1379

31 Kollef MH, Levy NT, Ahrens TS et al. The use of continuous i.v. sedation is associated with prolongation of mechanical ventilation. Chest 1998; 114 : $541-548$

32 Burns AM, Shelly MP, Park GR. The use of sedative agents in critically ill patients. Drugs 1992; 43: 507-515

33 Shelly MP. Sedation, where are we now? Intensive Care Med 1999; 25: $137-139$

34 Ely EW, Inouye SK, Bernard GR et al. Delirium in mechanically ventilated patients: validity and reliability of the confusion assessment method for the intensive care unit (CAM-ICU). JAMA 2001; 286: $2703-2710$

35 Hurel $D$, Loirat $P$, Saulnier $F$ et al. Quality of life 6 months after intensive care: results of a prospective multicenter study using a generic health status scale and a satisfaction scale. Intensive Care Med 1997; 23: 331 - 337

36 Granja C, Lopes A, Moreira S et al. Patients' recollections of experiences in the intensive care unit may affect their quality of life. Crit Care 2005; 9: R96-109

37 Kress JP, Pohlman AS, Alverdy J et al. The impact of morbid obesity on oxygen cost of breathing (VO(2RESP)) at rest. Am J Respir Crit Care Med 1999; 160: 883-886

38 Epstein SK, Ciubotaru RL. Independent effects of etiology of failure and time to reintubation on outcome for patients failing extubation. Am J Respir Crit Care Med 1998; 158: 489-493

39 Schönhofer B. Predictors of weanability. Monaldi Arch Chest Dis 2000; 55: 339-344

40 Milic-Emili J. Is weaning an art or a science? Am Rev Respir Dis 1986; 134: $1107-1108$

41 Brochard L, Rauss A, Benito $S$ et al. Comparison of three methods of gradual withdrawal from ventilatory support during weaning from mechanical ventilation. Am J Respir Crit Care Med 1994; 150: 896 903

42 Ely EW, Baker AM, Dunagan DP et al. Effect on the duration of mechanical ventilation of identifying patients capable of breathing spontaneously. N Engl J Med 1996; 335: 1864 - 1869

43 Esteban A, Alia I, Tobin MJ et al. Effect of spontaneous breathing trial duration on outcome of attempts to discontinue mechanical ventilation. Spanish Lung Failure Collaborative Group. Am J Respir Crit Care Med 1999; 159: $512-518$

44 Brochard L, Harf A, Lorino $\mathrm{H}$ et al. Inspiratory pressure support prevents diaphragmatic fatigue during weaning from mechanical ventilation. Am Rev Respir Dis 1989; 139: 513-521

45 Flick GR, Bellamy PE, Simmons DH. Diaphragmatic contraction during assisted mechanical ventilation. Chest 1989; 96: 130-135

46 Imsand $C$, Feihl $F$, Perret $C$ et al. Regulation of inspiratory neuromuscular output during synchronized intermittent mechanical ventilation. Anesthesiology 1994; 80: 13-22

47 Marini IJ, Capps JS, Culver BH. The inspiratory work of breathing during assisted mechanical ventilation. Chest 1985; 87: 612-618

48 Esteban A, Anzueto A, Frutos F et al. Characteristics and outcomes in adult patients receiving mechanical ventilation: a 28-day international study. JAMA 2002; 287: 345-355

49 Marini JJ, Smith TC, Lamb VJ. External work output and force generation during synchronized intermittent mechanical ventilation. Effect of machine assistance on breathing effort. Am Rev Respir Dis 1988; 138: $1169-1179$

50 Gibney RT, Wilson RS, Pontoppidan H. Comparison of work of breathing on high gas flow and demand valve continuous positive airway pressure systems. Chest 1982; 82: $692-695$

51 MacIntyre NR. Respiratory function during pressure support ventilation. Chest 1986; 89: 677-683

52 Jubran A, Graaf WVd, Tobin M. Variabilty of patient ventilator interaction with pressure support ventilation in patients with chronic obstructive pulmonary disease. Am J Respir Crit Care Med 1995; 1995: $129-136$ 
53 Laier-Groeneveld G, Rasche K, Weyland W et al. The oxygen cost of breathing in patients with chronic ventilatory failure. Am Rev Respir Dis 1992; 145: A155-A155

54 Schönhofer B, Euteneuer S, Nava S et al. Survival of mechanically ventilated patients admitted to a specialised weaning centre. Intensive Care Med 2002; 28: 908-916

55 Schönhofer B, Sonneborn M, Haidl P et al. Comparison of two different modes for noninvasive mechanical ventilation in chronic respiratory failure: volume versus pressure controlled device. Eur Respir J 1997; 10: $184-191$

56 Sassoon CS, Caiozzo VJ, Manka A et al. Altered diaphragm contractile properties with controlled mechanical ventilation. J Appl Physiol 2002; 92: 2585-2595

57 Vassilakopoulos T, Petrof BJ. Ventilator-induced diaphragmatic dysfunction. Am J Respir Crit Care Med 2004; 169: 336 - 341

58 Sassoon CS, Zhu E, Caiozzo VJ. Assist-control mechanical ventilation attenuates ventilator-induced diaphragmatic dysfunction. Am J Respir Crit Care Med 2004; 170: 626-632

59 Shapiro M, Wilson RK, Casar G et al. Work of breathing through different sized endotracheal tubes. Crit Care Med 1986; 14: 1028-1031

60 Boque MC, Gualis B, Sandiumenge A et al. Endotracheal tube intraluminal diameter narrowing after mechanical ventilation: use of acoustic reflectometry. Intensive Care Med 2004; 30: 2204-2209

61 Maeda Y, Fujino Y, Uchiyama A et al. Does the tube-compensation function of two modern mechanical ventilators provide effective work of breathing relief? Crit Care 2003; 7: R92 - 97

62 Epstein SK. Anatomy and physiology of tracheostomy. Respir Care 2005; 50: 476-482

63 Dulguerov P, Gysin C, Perneger TV et al. Percutaneous or surgical tracheostomy: a meta-analysis. Crit Care Med 1999; 27: 1617-1625

64 Rumbak MJ, Newton M, Truncale $T$ et al. A prospective, randomized, study comparing early percutaneous dilational tracheotomy to prolonged translaryngeal intubation (delayed tracheotomy) in critically ill medical patients. Crit Care Med 2004; 32: 1689- 1694

65 Antonelli M, Michetti V, Palma A Di et al. Percutaneous translaryngeal versus surgical tracheostomy: A randomized trial with 1 -yr doubleblind follow-up. Crit Care Med 2005; 33: 1015-1020

66 Heffner JE, Hess D. Tracheostomy management in the chronically ventilated patient. Clin Chest Med 2001; 22: 55-69

67 Cox CE, Carson SS, Holmes GM et al. Increase in tracheostomy for prolonged mechanical ventilation in North Carolina, 1993-2002. Crit Care Med 2004; 32: 2219-2226

68 Rosenblüh J, Schönhofer B, Kemper P et al. Bedeutung von Platzhaltern tracheotomierter Patienten während der Entwöhnungsphase nach Langzeitbeatmung. Med Klin 1994; 89: S61-63

69 Vitacca M, Vianello A, Colombo D et al. Comparison of two methods for weaning patients with chronic obstructive pulmonary disease requiring mechanical ventilation for more than 15 days. Am J Respir Crit Care Med 2001; 164: 225 - 230

70 Kress JP, Pohlman AS, O'Connor MF et al. Daily interruption of sedative infusion in critically il patients undergoing mechanical ventilation. N Engl J Med 2000; 342: 1471 - 1477

71 Krishnan JA, Moore D, Robeson C et al. A prospective, controlled trial of a protocol-based strategy to discontinue mechanical ventilation. Am J Respir Crit Care Med 2004; 169: 673 - 678

72 Hebert PC, Wells G, Blajchman MA et al. A multicenter, randomized, controlled clinical trial of transfusion requirements in critical care. Transfusion Requirements in Critical Care Investigators, Canadian Critical Care Trials Group. N Engl J Med 1999; 340: 409-417

73 Schönhofer B, Geibel M, Stickeler P et al. Endoscopic placement of a tracheal oxygen catheter: a new technique. Intensive Care Med 1997; 23: 445- 449

74 Schönhofer B, Köhler D. Stellenwert von oral appliziertem retardiertem Morphin zur Therapie des schwergradigen Lungenemphysems vom Pink-Puffer-Typ. Dtsch Med Wochenschr 1998; 123: 14331438

75 Celli BR, Cote CG, Marin JM et al. The body-mass index, airflow obstruction, dyspnea, and exercise capacity index in chronic obstructive pulmonary disease. N Engl J Med 2004; 350: 1005 - 1012

76 Bassili HR, Deitel M. Nutritional support in long term intensive care with special reference to ventilator patients: a review. Can Anaesth Soc 1991; 28: $17-21$
77 Bolton $C F$, Breuer AC. Critical illness polyneuropathy. Muscle Nerve 1999; 22: 419-424

78 Burdet L, Muralt B de, Schutz Y et al. Administration of growth hormone to underweight patients with chronic obstructive pulmonary disease. A prospective randomized controlled study. Am J Respir Crit Care Med 1997; 156: 1800- 1806

79 Ferreira I, Verreschi I, Nery L et al. The influence of 6 months of oral anabolic steroids on body mass and respiratory muscles in undernourished COPD patients. Chest 1998; 114: 19-28

80 Schols AMW, Soeters PB, Mostert R et al. Physiologic effects of nutritional support and anabolic steroids in patients with chronic obstructive pulmonary disease. A placebo-controlled randomized trial. Am J Respir Crit Care Med 1995; 152: 1268 - 1274

81 Barr J, Hecht M, Flavin KE et al. Outcomes in Critically Ill Patients Before and After the Implementation of an Evidence-Based Nutritional Management Protocol. Chest 2004; 125: 1446-1457

82 Karg O, Bonnet R, Magnussen $\mathrm{H}$ et al. Respiratory Therapist - Atmungstherapeut - Einführung eines neuen Berufsbildes. Pneumologie 2004; 58: $854-857$

83 Thomason JW, Shintani A, Peterson JF et al. Intensive care unit delirium is an independent predictor of longer hospital stay: a prospective analysis of 261 non-ventilated patients. Crit Care 2005; 9: R375381

84 Granberg Axell AI, Malmros CW, Bergbom IL et al. Intensive care unit syndrome/delirium is associated with anemia, drug therapy and duration of ventilation treatment. Acta Anaesthesiol Scand 2002; 46: $726-731$

85 Ramsay MA, Savege TM, Simpson BR et al. Controlled sedation with alphaxalone-alphadolone. Br Med J 1974; 2: 656-659

86 Nava S, Ambrosino N, Clini E et al. Noninvasive mechanical ventilation in the weaning of patients with respiratory failure due to chronic obstructive pulmonary disease. A randomized, controlled trial. Ann Intern Med 1998; 128: $721-728$

87 Ferrer M, Esquinas A, Arancibia F et al. Noninvasive ventilation during persistent weaning failure: a randomized controlled trial. Am J Respir Crit Care Med 2003; 168: 70-76

88 Kilger E, Briegel J, Haller $M$ et al. Effects of noninvasive positive pressure ventilatory support in non-COPD patients with acute respiratory insufficiency after early extubation. Intensive Care Med 1999; 25 : $1374-1380$

89 Carlucci A, Gregoretti C, Squadrone V et al. Preventive use of non-invasive mechanical ventilation to avoid post-extubation respiratory failure: a randomised controlled study. Eur Respir J 2001; 18 suppl 33: $306-306$

90 Hilbert G, Gruson D, Portel L et al. Noninvasive pressure support ventilation in COPD patients with postextubation hypercapnic respiratory insufficiency. Eur Respir J 1998; 11: 1349-1353

91 Nava S, Gregoretti C, Fanfulla $F$ et al. Noninvasive ventilation to prevent respiratory failure after extubation in high-risk patients. Crit Care Med 2005; 33: 2465-2470

92 Ferrer $M$, Valencia M, Nicolas JM et al. Early noninvasive ventilation averts extubation failure in patients at risk: a randomized trial. Am J Respir Crit Care Med 2006; 173: 164 - 170

93 Keenan SP, Powers C, McCormack DG et al. Noninvasive positive-pressure ventilation for postextubation respiratory distress: a randomized controlled trial. JAMA 2002; 287: 3238 - 3244

94 Esteban A, Frutos-Vivar F, Ferguson ND et al. Noninvasive positivepressure ventilation for respiratory failure after extubation. $\mathrm{N}$ Engl J Med 2004; 350: $2452-2460$

95 Schönhofer B, Wagner TOF. Ort der maschinellen Beatmung im Beatmungszentrum - Intensivstation, Intermediate care oder spezialisierte Normalstation. Pneumologie 2006; 60: 376- 382

96 Chatila W, Kreimer DT, Criner GJ. Quality of life in survivors of prolonged mechanical ventilatory support. Crit Care Med 2001; 29: $737-742$

97 Hopkins RO, Weaver LK, Collingridge D et al. Two-year cognitive, emotional, and quality-of-life outcomes in acute respiratory distress syndrome. Am J Respir Crit Care Med 2005; 171: 340-347

98 Euteneuer S, Windisch $W$, Suchi S et al. Health-related quality of life in patients with chronic respiratory failure after long-term mechanical ventilation. Respir Med 2006; 100: 477-486

99 Stoller JK. Establishing Clinical Unweanability. Respir Care 1991; 36: $186-198$ 
100 Schönhofer B, Köhler D, Kutzer K. Ethische Betrachtungen zur Beatmungsmedizin unter besonderer Berücksichtigung des Lebensendes. Pneumologie 2006; 60: 408 - 416

101 Cook D, Rocker G, Marshall J et al. Withdrawal of mechanical ventilation in anticipation of death in the intensive care unit. N Engl J Med 2003; 349: $1123-1132$

102 Gianakos D. Terminal weaning. Chest 1995; 108: 1405-1406

103 Nava S, Zanotti E, Rubini F. Weaning and outcome from mechanical ventilation. Monaldi Arch Chest Dis 1994; 49: 530-532
104 Gracey DR, Naessens JM, Viggiano RW et al. Outcome of patients cared for in a ventilator-dependent unit in a general hospital. Chest 1995; 107: 494-499

105 Scheinhorn DJ, Artinian BM, Catlin JL. Weaning from prolonged mechanical ventilation. The experience at a regional weaning center. Chest 1994; 105: 534-539

106 Bagley PH, Cooney E. A community-based regional ventilator weaning unit: development and outcomes. Chest 1997; 111: 1024-1029 